\title{
Constructing Optimal Visualization Metaphor of Fuzzy Cognitive Maps on the Basis of Formalized Cognitive Clarity Criteria
}

\author{
A.G. Podvesovskiii,A, R.A. Isaev²,A \\ Bryansk State Technical University \\ ${ }^{1}$ ORCID: 0000-0002-1118-3266, apodv@tu-bryansk.ru \\ 2 ORCID: oooo-0003-3263-4051, ruslan-isaev-32@yandex.ru
}

\begin{abstract}
The paper presents continuation of research in the field of constructing a visualization metaphor of cognitive models based on fuzzy cognitive maps. Development of this metaphor in the direction of automating the construction of a visual image of a fuzzy cognitive map, which is optimal from the point of view of cognitive clarity criteria defined in the previous part of the study, is proposed. To this end, methods have been developed to formalize several nontrivial criteria of cognitive clarity. Also, a decision rule has been introduced for choosing the optimal visual image. An example of applying the proposed metaphor, confirming its efficiency and effectiveness, is given.
\end{abstract}

Keywords: fuzzy cognitive map, graph visualization, cognitive clarity, visualization metaphor.

\section{Introduction}

This paper continues a series of publications of authors' research materials in the field of visualization of cognitive models based on fuzzy cognitive maps (FCM). A FCM reflects researcher's subjective idea of a system in the form of a set of semantic categories (called factors or concepts) and a set of causal relationships between them [1,2]. Thus, a FCM can be graphically represented in the form of a weighted directed graph, the vertices of which correspond to concepts, and edges - to cause-and-effect relationships.

One of the conditions for effective work with a cognitive model is ensuring its visual representation. In [3], the authors proposed an approach to FCM visualization based on using the concept of visualization metaphor and its two components - spatial metaphor and representation metaphor [4]. FCM visualization metaphor is based on graph visualization algorithms $[5,6]$ and cognitive clarity concept, which characterizes the ease of intuitive understanding of information [7] and takes into account the problem of human's limited cognitive abilities when reading graphs (a detailed analysis of this problem can be found, for example, in [8]). Thus, a link has been discovered between the quality of FCM visualization metaphor and the level of cognitive clarity of the resulting visual image: the higher the level of cognitive clarity provided by the visualization metaphor, the simpler is the process of expert understanding of the cognitive model in its visual analysis. To assess the level of cognitive clarity, a set of criteria is proposed. It is concluded that cognitive clarity criteria are the means of the most natural evaluation of visualization metaphor quality.

The present work is devoted to the development of FCM visualization metaphor in the direction of automating the construction of FCM visual image, which is optimal in terms of cognitive clarity criteria. The previous part of the study focused on the representation metaphor, whereas this work focuses on the spatial metaphor, which is the basic component of the visualization metaphor and serves as the foundation for the subsequent formation of the representation metaphor. 


\section{Development of FCM visualization metaphor: basic concept}

In [3], it was noted that many of cognitive clarity criteria contradict each other, and it is impossible in the general case to ensure that FCM visual image meets all the criteria at the same time from an algorithmic point of view.

However, a number of features of FCM visualization process are noteworthy:

1) existing graph visualization algorithms $[5,6]$ ensure the formation of sufficiently acceptable (from the point of view of individual cognitive clarity criteria) visual images of an FCM;

2) building FCM visual image using such algorithms does not require large computational and time resources. Thus, it is possible to generate a large number of visual images of the selected FCM within a feasible period of time (including application of parallel computing technologies);

3) with a number of constructed visual images of an FCM available, in the general case, an image can be chosen from them that most fully meets cognitive clarity criteria.

These features formed the basis for the development of FCM visualization metaphor to ensure the construction of FCM visual image that is optimal in terms of cognitive clarity criteria. The proposed FCM visualization algorithm using metaphor is shown in Fig. 1.

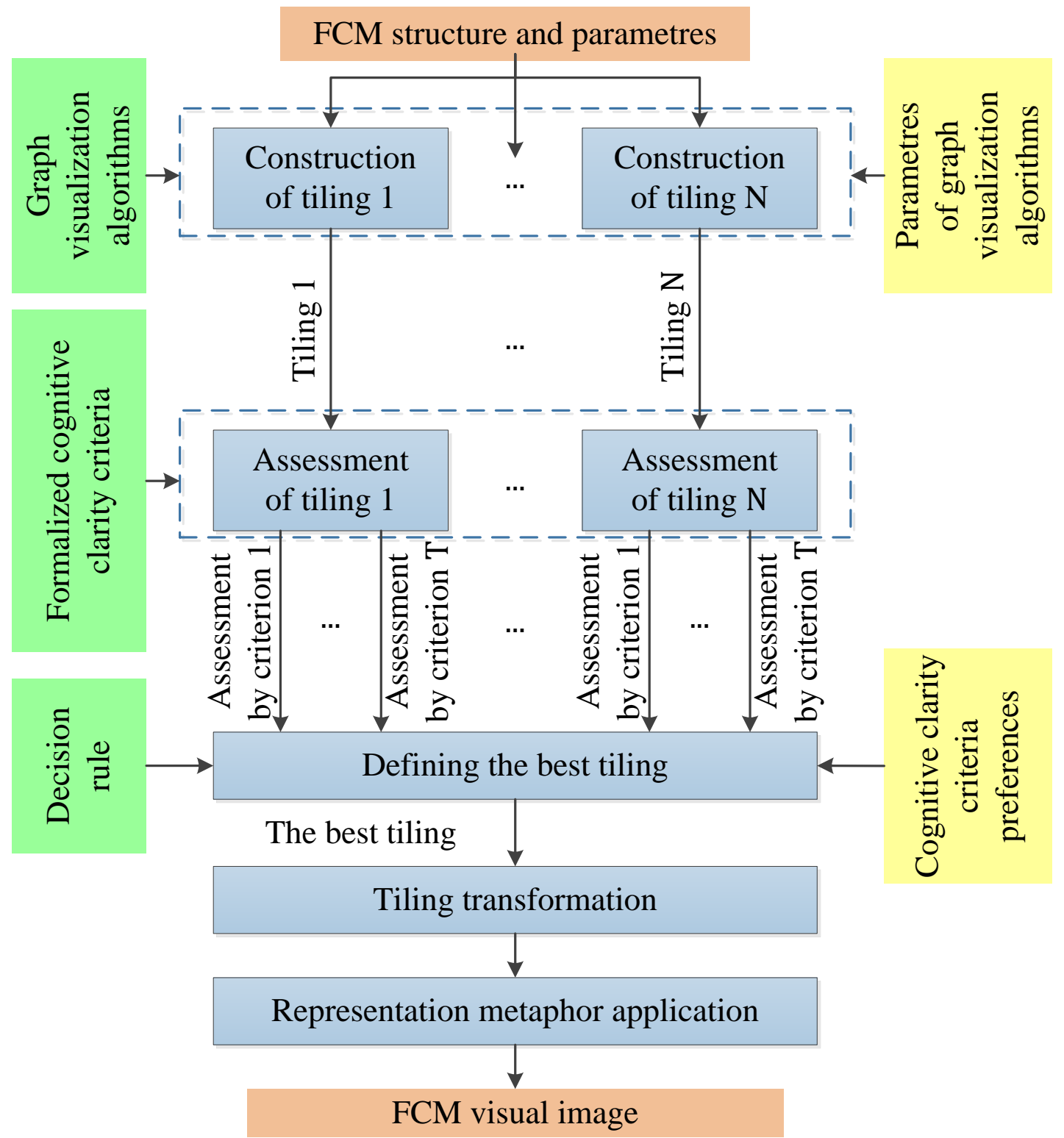

Fig. 1. FCM visualization algorithm using metaphor 
Let us describe the stages of this algorithm.

At the first stage, graph visualization algorithms are applied to construct $N$ different tilings of a cognitive graph (where number $N$ can be quite large and depends on the system performance and the allowed time spent at this stage). Graph tiling refers to the collection of coordinates of all its vertices and edges. Thus, tiling uniquely sets the location of all elements of the graph in space (in the two-dimensional case under consideration, on the plane).

The possibility of obtaining a large number of different tilings of the same graph is based on the following factors, as well as their combinations:

1) at this stage, a set of different graph visualization algorithms can be applied, those operation is based on various principles and therefore leads to the formation of different tilings with the same input data;

2) as a rule, graph visualization algorithms provide for a number of customizable parameters (e.g., formulas coefficients), changing of which affects the results of the algorithms, i.e. the resulting tilings;

3) as a rule, graph visualization algorithms use as input data the initial coordinates of vertices and edges generated randomly at each new start of the algorithm. Thus, stochastic elements are introduced into the results of their work.

It should be noted that tilings built at this stage, due to their large number, are not displayed on the screen, but just stored in RAM.

The second stage of the algorithm involves assessing the degrees of conformity of the generated tilings to the criteria of cognitive clarity. Note that these criteria are formulated at a qualitative level using a natural language. At the same time, the proposed metaphor, that targets the automation of the construction of an optimal FCM visual image, implies the implementation of all the main stages of the algorithm without human participation. Therefore, the stage of assessment of the resulting tiles according to the cognitive clarity criteria needs the algorithm to be designed. For this purpose, it is necessary to obtain a formalized representation of these criteria. This issue will be discussed in more detail below.

At the third stage of the algorithm, on the basis of the obtained criterial estimates of the tilings and previously specified priorities of the cognitive clarity criteria, an optimal tiling is selected. In this case, it is necessary to apply decision rules that model various forms of compromise among the criteria. One of the admissible rules is proposed further in this paper.

The goal of the transformation stage of the selected optimal tiling is to further increase its cognitive clarity by performing one or more of the following types of operations: rotation by a certain angle, reflection relative to the horizontal or vertical axis, compression or extension along a certain direction. Thus, this stage performs the function of post-processing of the resulting tile and is generally not mandatory.

Finally, the last stage of the algorithm involves displaying FCM visual image based on the selected optimal tiling. Herewith, as a rule, representation metaphor is applied that corresponds to the current stage of construction or analysis of a cognitive model.

Obviously, the efficiency of the proposed algorithm depends on number of the processed tilings $N$ : the more tilings have been generated, the higher the probability is that a tiling with a high level of cognitive clarity will be found among them. At the same time, in practice, the value of $N$ must be limited in order to satisfy the specified time constraints on the process of constructing a visual image of an FCM.

It should be also noted that the stages of the algorithm surrounded by dashed lines (i.e., the construction of tilings and their assessment by criteria) can be performed simultaneously. In the case of using parallel computing technologies, this can significantly increase number $N$, which will lead to an increase in the efficiency of the algorithm.

\section{Methods of cognitive clarity criteria formalization}

We define the formalization of a certain cognitive clarity criterion as the developing methods, techniques and algorithms that allow determining a numerical score for a visual image of an 
arbitrary cognitive map characterizing the extent to which this image complies with the selected criterion. Formalization of most criteria (e.g. such as minimizing edge lengths, minimizing edge crossing, minimizing the number of curved edges) is trivial, and its description is of no interest as it reduces to solving simple problems of computational geometry. Let us consider possible ways of formalizing several nontrivial cognitive clarity criteria.

\subsection{Optimizing edge directions}

This criterion is based on the observation that laying out edges in the directions "from top to bottom" and "from left to right" helps to accelerate "reading" of a FCM in comparison with the orientation of edges in the opposite directions. We shall call the directions that facilitate faster "reading" of FCMs as well as edges having such directions convenient. As an example, we can compare two visual images of a cognitive graph in Figure 2.

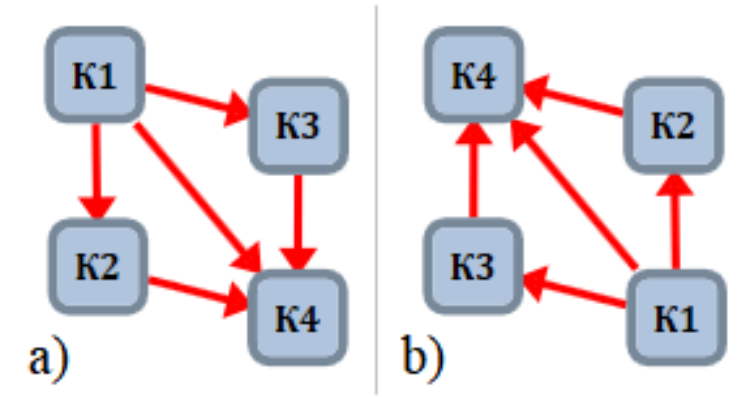

Fig. 2. Examples of visual images with convenient (a) and not convenient (b) directions of edges

Apparently, convenient directions coincide with the direction of reading, adopted in a particular language culture. Therefore, other conditions being equal, preference should be given to visual images containing a greater number of convenient edges. It should be borne in mind that the described property is inherently fuzzy. So, edge orientation "from top to bottom" and "from right to left" can be considered partially convenient, since one of the usual directions of reading is preserved. Therefore, the mathematical apparatus of the fuzzy set theory can be used to formalize the criterion in question.

Let $A$ be a fuzzy set formalizing the concept of a "convenient edge direction". In order to set its membership function, let us define the edge direction as angle $\alpha$ between the vector drawn from the beginning of the edge to its end and the positive direction of the horizontal axis OX.

Then membership function $\mu_{A}(\alpha)$ must satisfy the following requirements:
1) $\mu_{A}(\alpha)=1$ when $-\frac{\pi}{2} \leq \alpha \leq 0$;
2) $\mu_{A}(\alpha)=0$ when $\frac{\pi}{2} \leq \alpha \leq \pi$;
3) $0<\mu_{A}(\alpha)<1_{\text {when }} 0<\alpha<\frac{\pi}{2}$ и $-\pi<\alpha<-\frac{\pi}{2}$;
4) increases monotonically on the interval $-\pi<\alpha<-\frac{\pi}{2}$;
5) decreases monotonically on the interval $0<\alpha<\frac{\pi}{2}$. 
Given these requirements, $\mu_{A}(\alpha)=\cos (\alpha)$ can be accepted on the interval $0<\alpha<\frac{\pi}{2}$, and $\mu_{A}(\alpha)=-\sin (\alpha)$. can be accepted on the interval $-\pi<\alpha<-\frac{\pi}{2}$.

Having determined for each FCM edge the degree of its membership to set $A$, we can obtain a value characterizing the overall score of the entire visual image by this criterion - for example, as the average value of membership degrees of all edges.

Influence intensities should also be taken into account in the final assessment, since providing convenient directions for more significant influences is more important than for less significant ones. Therefore, absolute values of influence intensities can be used as weighting coefficients and membership values of the corresponding edges can be multiplied by them when calculating the average value.

\subsection{Maximizing unidirectionality of consecutive edges}

This criterion is based on the idea that "reading" a FCM will be faster if gaze direction has to be changed as little as possible during the process of viewing paths and cycles of a graph.

We will call two edges consecutive if one of them enters the vertex from which the other one starts. Thus, any path and cycle of a graph consists of pairs of consecutive edges. Therefore, in accordance with this criterion, preference should be given to visual images with a greater number of pairs of consecutive edges depicted unidirectionally. For example, let us compare two visual images of a fragment of some FCM (Fig. 3).

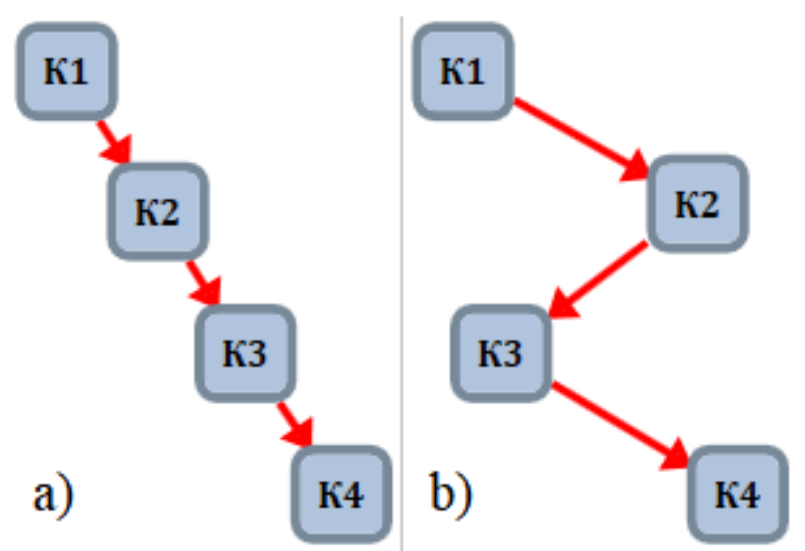

Fig. 3. Examples of visual images with unidirectional (a) and bidirectional (b) consecutive edges

Obviously, the unidirectional property is fuzzy. Suppose $B$ is a fuzzy set formalizing the concept of unidirectional edges. The membership degree of a pair of consecutive edges to set $B$ is determined by angle $\beta \in[0, \pi]$ between these edges. We assume that changing gaze direction by 90 degrees or more slows down the process of viewing the path in the graph significantly.

Accordingly, the following requirements are imposed on the membership function $\mu_{B}(\beta)$ :

1) $\mu_{B}(\beta)=1$ when $\beta=0$;

2) $\mu_{B}(\beta)=0$ when $\beta \geq \frac{\pi}{2}$;

3) $0<\mu_{B}(\beta)<1$ and decreases monotonically when $0<\beta<\frac{\pi}{2}$. 
Given these requirements, on the interval $0<\beta<\frac{\pi}{2}$ we accept $\mu_{B}(\beta)=\cos (\beta)$.

By analogy with the previous criterion, the score of the entire visual image by the criterion under study can be found as the average value of membership degrees of all pairs of consecutive edges to set $B$. Influence intensities can also be taken into account in a similar way.

\subsection{Maximizing graph symmetry}

Due to the fact that FCM structure reflects the structure of a simulated system it is important to ensure the symmetry of FCM visual image to increase its cognitive clarity. Thus, symmetries of a graph image help to detect symmetries inherent in the system itself.

Let us consider various aspects of determining degree of image symmetry in relation to FCM visual image.

Firstly, the following types of symmetries are the simplest to perceive and, therefore, of greatest practical interest:

1) axial symmetry with respect to the horizontal axis of an image;

2) axial symmetry with respect to the vertical axis of an image;

3) central symmetry with respect to the geometric center of an image.

Secondly, in the case of an FCM, as well as any digraph, the following levels of symmetry can be distinguished (Fig. 4 considers the case of symmetry about the vertical axis):

1) lack of symmetry at the level of any elements of the graph (Fig. 4, a);

2) at the level of vertices excluding edges (Fig. 4, b);

3) at the level of edges excluding their directions (Fig. 4, c);

4) at the level of edges including their directions (Fig. 4, d).

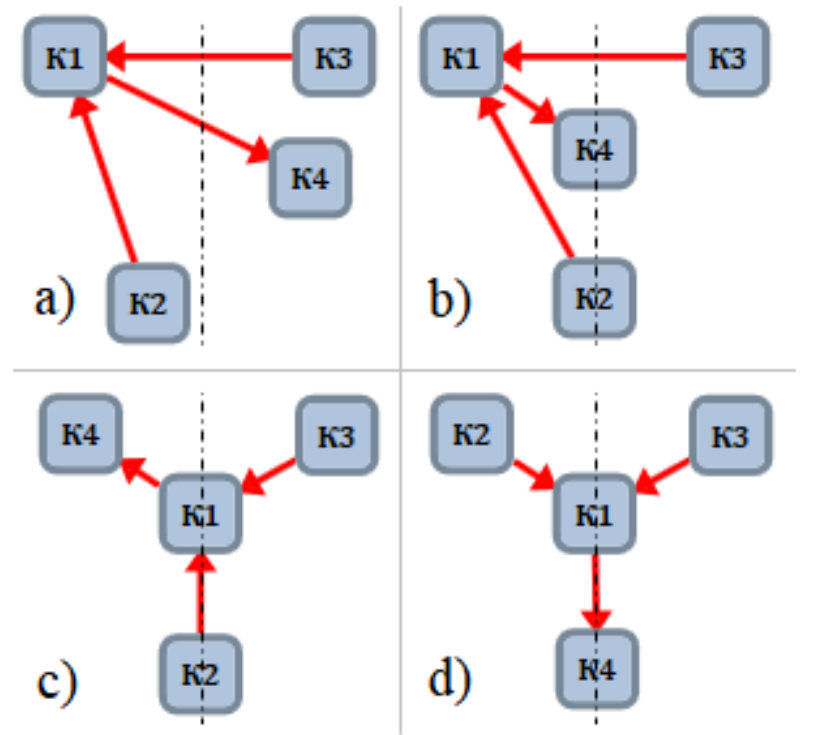

Fig. 4. Examples of different symmetry levels of FCM visual image (about the vertical axis)

The above example allows for the conclusion that symmetry at the vertex level does not bring any tangible effect to increasing cognitive clarity of an FCM visual image. Thus, only symmetry at the level of edges is of practical interest.

Thirdly, it is obvious that in addition to strict symmetry (Fig. 5, a), we can also speak of approximate symmetry (Fig. 5, b), which can be represented as a certain deviation from the strict one. 


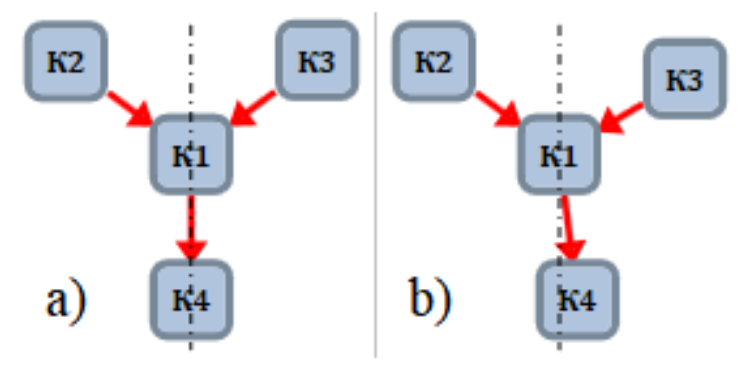

Fig. 5. Examples of strict (a) and approximate (b) symmetry of FCM visual image

With this in mind, the degree of symmetry of an FCM visual image can be defined as a measure of its proximity to a strictly symmetric image. Thus, it is necessary to develop an algorithm that is able to determine the degree of symmetry for an arbitrary image taking into account a given type and level of symmetry.

The main idea of the proposed algorithm is as follows. For each element of an FCM visual image, the position of its "reflection" relative to a given axis or center is calculated. Next, for each of the "reflections", the element closest to it (in the sense of the chosen metric, for example, Euclidean distance) is selected from among all the elements of the image. Distances (in the selected metric) from all "reflections" to their nearest elements are added up. The resulting value characterizes the degree of symmetry of the visual image in question and has the following properties:

1) it is equal to $o$ if the image has strict symmetry of a given (or stronger) level and type;

2) it is greater than $o$ in all other cases;

3) it increases as the image becomes less and less symmetrical;

4) it does not have an upper bound since there is no "maximally asymmetric" image.

\section{Development of a decision rule for choosing an opti- mal FCM tiling}

In the context of the problem under consideration, of primary interest is the class of decision rules based on various types of criteria aggregations, primarily, sum and product ones. At the same time, there is reason to believe that the structure of relationships among cognitive clarity criteria is quite complex and is characterized by the following features:

1) criteria may exist that determine quality of a metaphor not separately but in combination with some other criteria;

2) in the whole set of criteria, there may be several "bundles of criteria" affecting metaphor quality independently of each other.

To formalize the described assumption, we shall accept that set of criteria $K=\left\{k_{1}, \ldots, k_{n}\right\}$ can be divided into disjoint subsets $G_{1}, \ldots, G_{m}$. Further, we will also assume that FCM visual image scores by all criteria take their values in the interval $[0,1]$.

For each criteria subset $G_{i}$, we shall introduce value $g_{i} \in[0 ; 1]$ - visual image score for this subset. We shall specify the following requirements for such a score:

1) if the image score by at least one criterion from subset $G_{i}$ is o, then $g_{i}=0$;

2) $g_{i}=1$ if and only if the image score by all criteria from subset $G_{i}$ is 1 ;

3) if the image score according to all criteria from subset $G_{i}$ is $a$, then $g_{i}=a$ (idempotency).

One of the operations meeting the specified requirements is a weighted product aggregation:

$$
g_{i}=\prod_{j=1}^{l} m_{j}^{w_{j}}
$$


where ${ }^{m_{j}}$ is image score by the $j$-th criterion from $G i, w_{j} \in[0 ; 1]$ is relative importance of the $j$-th criterion within $G_{i}\left(w_{1}+\ldots+w_{l}=1\right), l$ is power of $G_{i}$.

To obtain final score, we shall apply weighted sum aggregation to the scores obtained for all subsets:

$$
F=\sum_{i=1}^{m} w_{g_{i}} g_{i}
$$

where ${ }^{w_{g_{i}} \in[0 ; 1]}-$ is relative importance of subset $G_{i}\left(w_{g_{1}}+\ldots+w_{g_{m}}=1\right)$.

Moreover, value $w_{g_{i}}$ can be interpreted as FCM visual image score that fully satisfies the subset of criteria $G_{i}$ and does not completely satisfy other subsets of criteria.

Thus, for the final score $F$, the following properties are guaranteed:

1) $F \in[0 ; 1]$;

2) $F=1$ if scores for all criteria are 1 ;

3) $F=\mathrm{o}$ if scores for all subsets of criteria $G_{1}, \ldots, G_{m}$ are o (i.e., at least one criterion scored with $\mathrm{O}$ is present in each subset).

It should be noted that since the proposed decision rule is based on the combined use of sum and product aggregations of criteria these types of aggregations are its "extreme" special cases. Thus, a sum aggregation type will be obtained if a separate subset $G_{1}, \ldots, G_{n}$ is assigned to each of the criteria $k_{1}, \ldots, k_{n}$. Assignment of all criteria to one subset $G_{1}$ will result in a product aggregation.

Selection and justification of parameters of the decision rule (the number of subsets of criteria, distribution of criteria by subsets, etc.) is the task of the analyst performing a visual analysis of an FCM. This problem should be solved from knowledge of the features of a particular stage of cognitive modeling (in particular, which cognitive clarity criteria are most significant at this stage), as well as involving analyst's intuition and experience. So, the relative importance of the subsets and criteria within the subsets can be set based on a pairwise comparison method.

\section{Experimental procedure}

The complex of algorithms providing the proposed development of FCM visualization metaphor was implemented within the framework of FCM visualization subsystem, which is part of IGLA DSS based on fuzzy cognitive models [9].

In order to verify the operability of the proposed development of FCM visualization metaphor, a series of experiments was carried out. Within its framework the following metaphor parameters were varied:

- graph visualization algorithms used;

- number of generated tilings $N$;

- system of preferences based on cognitive clarity criteria.

Based on the results of the series of experiments, the following conclusions have been made:

- all varied parameters influence the quality of the metaphor, i.e. the level of cognitive clarity of the resulting visual image;

- the most appropriate is the use of graph visualization algorithms ISOM and LinLog;

- an acceptable level of metaphor quality is achieved already when $N=100$, while an increase in $\mathrm{N}$ leads to an increase in time spent on the visualization process, which for large values of $\mathrm{N}$ may be unacceptable (note that the unit cost of processing one tiling is 0.03o.o9 seconds depending on the size of FCM; these data were obtained under the following conditions: i5-2450M processor, parallel computing technologies were not used); 
- the decision rule introduced in this paper allows for flexible control of the relative importance of cognitive clarity criteria and helps to determine the acceptable forms of compromise among them.

Let us consider in more detail conditions and results of one of the experiments.

An FCM of analysis and planning of software projects was selected as an FCM for which a visual image had to be built [10]. The choice of this FCM was determined by its relatively small size and simple structure, which positively affected the ease of interpretation of the visualization results obtained (and, thus, simplified the verification of the metaphor under study).

At the stage of constructing FCM tilings, ISOM and LinLog algorithms were implemented. A total of 100 tilings were generated. Assessment of the degree of compliance of the tilings with cognitive clarity criteria was performed using the proposed methods of formalizing these criteria.

The parameters of the decision rule were set as follows. The set of criteria was divided into two subsets: $G_{1}$ and $G_{2}$. At the same time, the following criteria were assigned to the subset $G_{1}$ :

- optimizing placement area (within the framework of this experiment, tiling had to be placed in a square-shaped area);

- minimizing edge lengths;

- unifying edge lengths;

- maximizing graph symmetry.

Subset $G_{2}$ included the rest of the criteria:

- optimizing edge directions;

- minimizing edge crossing;

- minimizing the number of curved edges;

- maximizing unidirectionality of consecutive edges;

- maximizing angles between incident edges.

The priorities of the subsets $G_{1}$ and $G_{2}$ were set equal to 0.3 and 0.7 , respectively. Criteria priorities within the subsets were distributed evenly, i.e., 0.25 and 0.2 for the criteria from $G_{1}$ and $G_{2}$, respectively.

The tiling shown in Fig. 6 was recognized the best of those generated (hereinafter we will refer to it as Tiling 1). For comparison, let us also consider two other tilings, randomly selected from among generated ones (Fig. 7-8), which we will refer to as 2 and 3, respectively.

Table 1 presents the results of tiling assessment according to the cognitive clarity criteria. Criteria scores of tilings are normalized to the range $[0,1]$ (normalization was carried out taking into account the scores of all 100 tilings obtained). Meanwhile, the initial requirement to minimize a number of criteria was taken into account. Thus, after normalization, all criteria must be maximized. 
Table 1. Results of assessment of generated tilings

\begin{tabular}{|l|l|l|l|}
\hline \multirow{2}{*}{ Criteria and subsets of criteria } & \multicolumn{2}{l}{ Tiling number } & 3 \\
\cline { 2 - 4 } & 1 & 2 & 3 \\
\hline Subset $\boldsymbol{G}_{\mathbf{1}}$ & $\mathbf{0 . 8 6 0}$ & 0.715 & 0.467 \\
\hline Optimizing placement area & 0.824 & $\mathbf{0 . 9 8 5}$ & 0.792 \\
\hline Minimizing edge lengths & $\mathbf{0 . 7 9 6}$ & 0.654 & 0.439 \\
\hline Unifying edge lengths & $\mathbf{0 . 9 5 2}$ & 0.696 & 0.299 \\
\hline Maximizing graph symmetry & $\mathbf{0 . 8 7 5}$ & 0.584 & 0.457 \\
\hline Subset $\boldsymbol{G}_{\mathbf{2}}$ & 0.749 & 0.410 & $\mathbf{0 . 7 7}$ \\
\hline Optimizing edge directions & $\mathbf{0 . 8 0 4}$ & 0.232 & 0.765 \\
\hline Minimizing edge crossing & $\mathbf{1}$ & 0.540 & $\mathbf{1}$ \\
\hline Minimizing the number of curved edges & $\mathbf{1}$ & 0.673 & 0.694 \\
\hline $\begin{array}{l}\text { Maximizing unidirectionality of consecutive } \\
\text { edges }\end{array}$ & 0.368 & 0.350 & $\mathbf{0 . 8 9 2}$ \\
\hline Maximizing angles between incident edges & $\mathbf{0 . 7 9 6}$ & 0.394 & 0.603 \\
\hline Final score & $\mathbf{0 . 7 8 2}$ & 0.502 & 0.685 \\
\hline
\end{tabular}

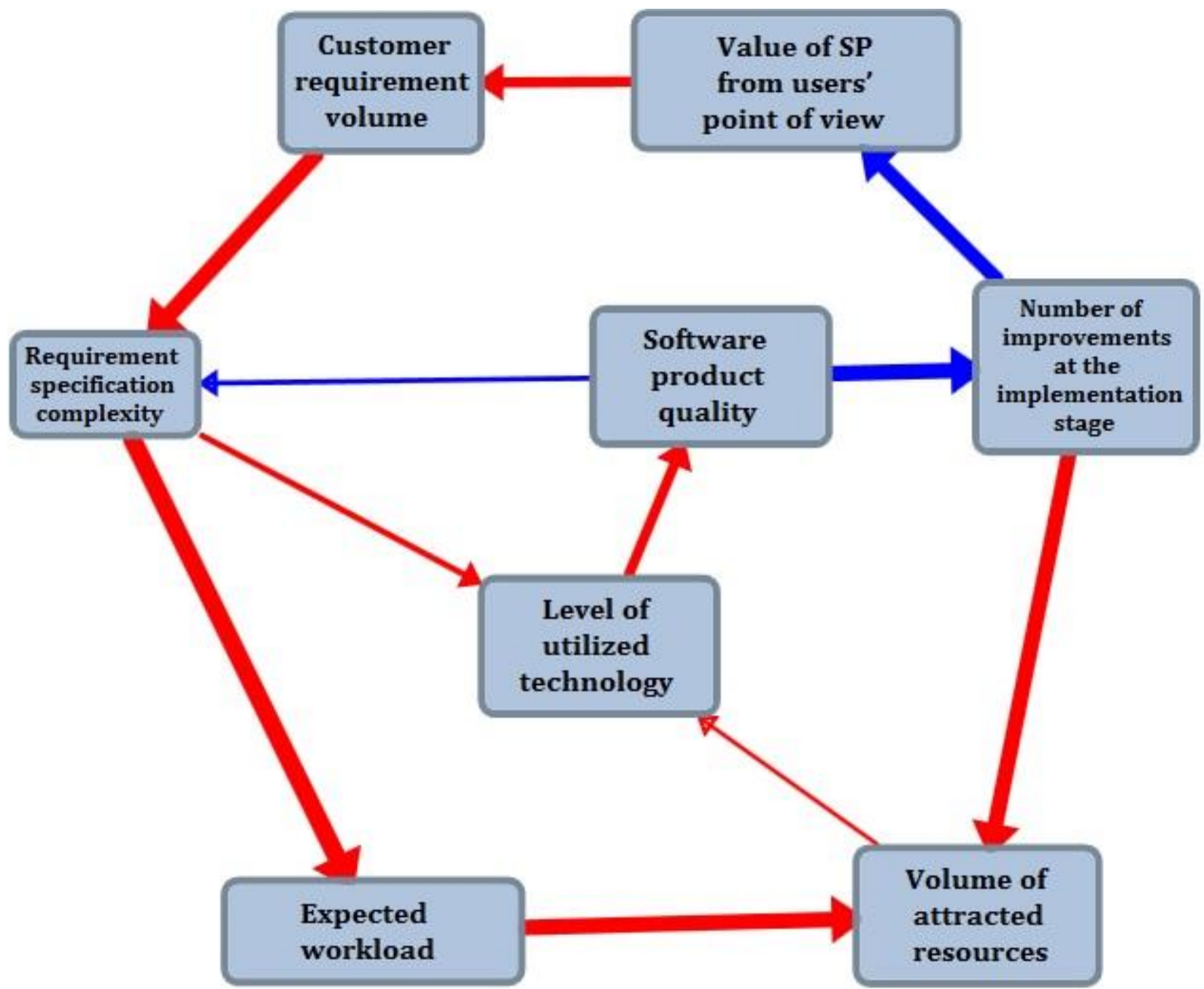

Fig. 6. Tiling 1 


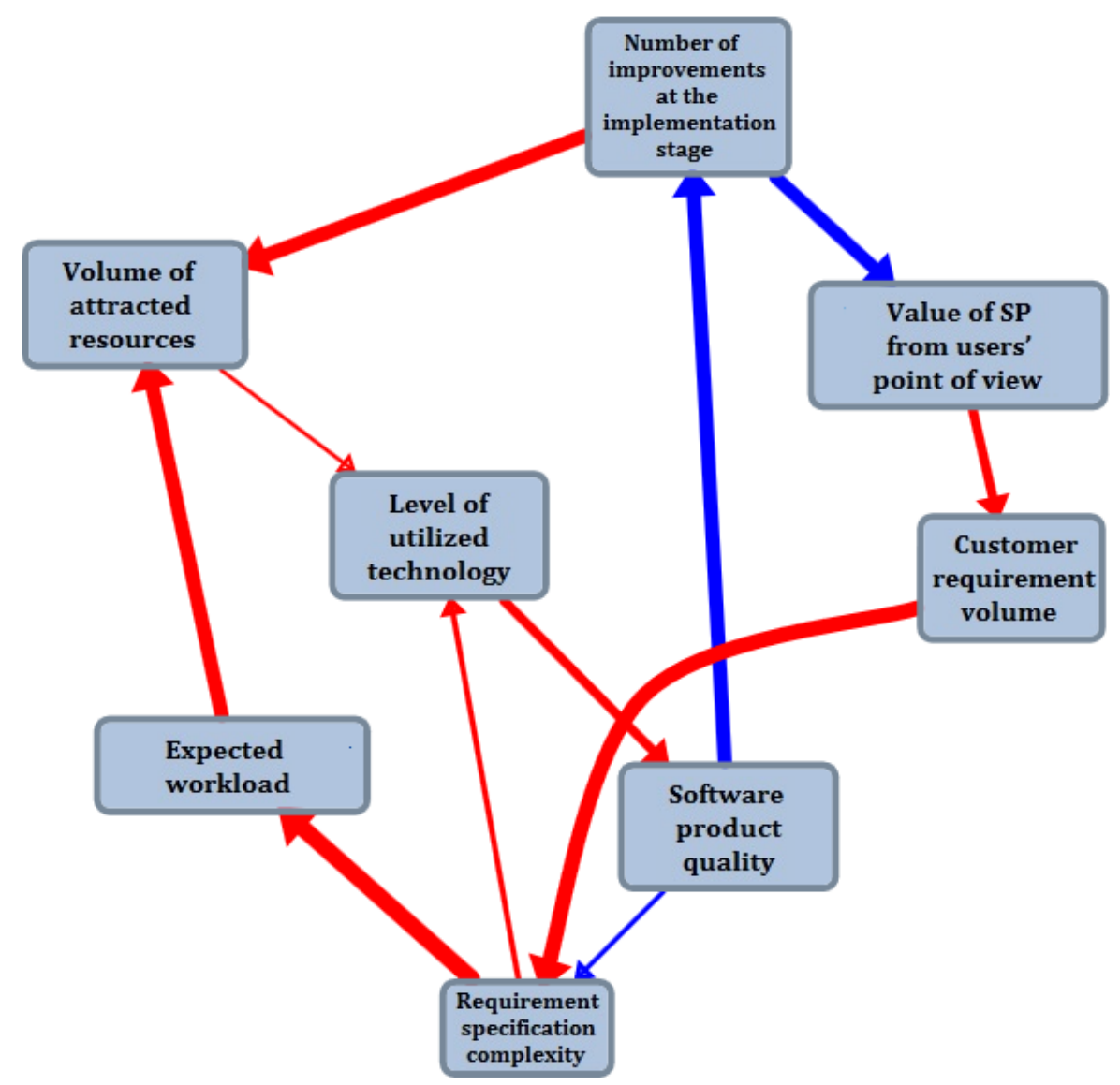

Fig. 7. Tiling 2

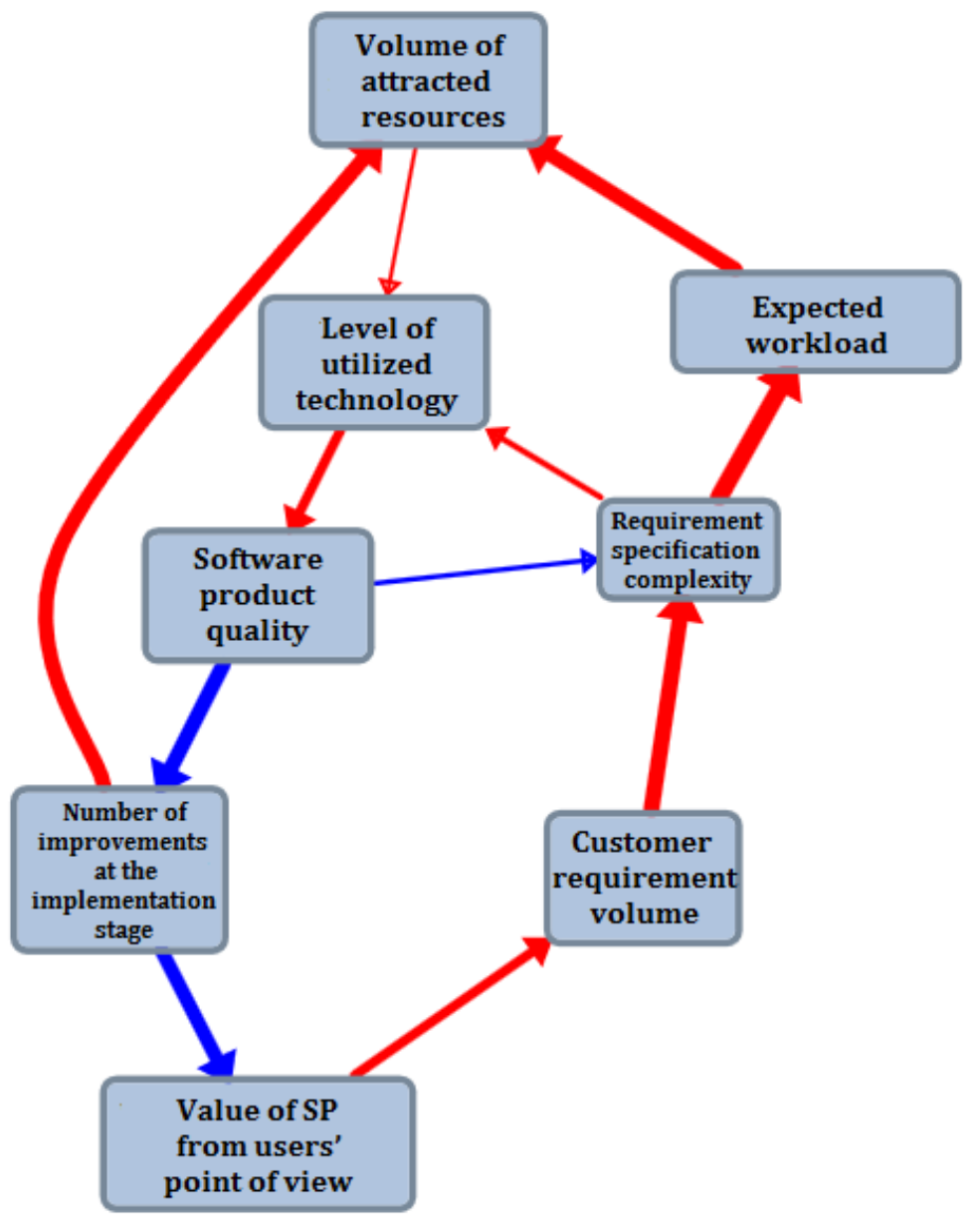

Fig. 8. Tiling 3 
Figure 9 presents the final version of FCM visual image obtained on the basis of Tiling 1 as a result of transformation operations performed on it. In this case, the following sequence of operations was performed: 90 degree clockwise rotation; mirror reflection with respect to the vertical axis of tiling; vertical compression of tiling by $30 \%$.

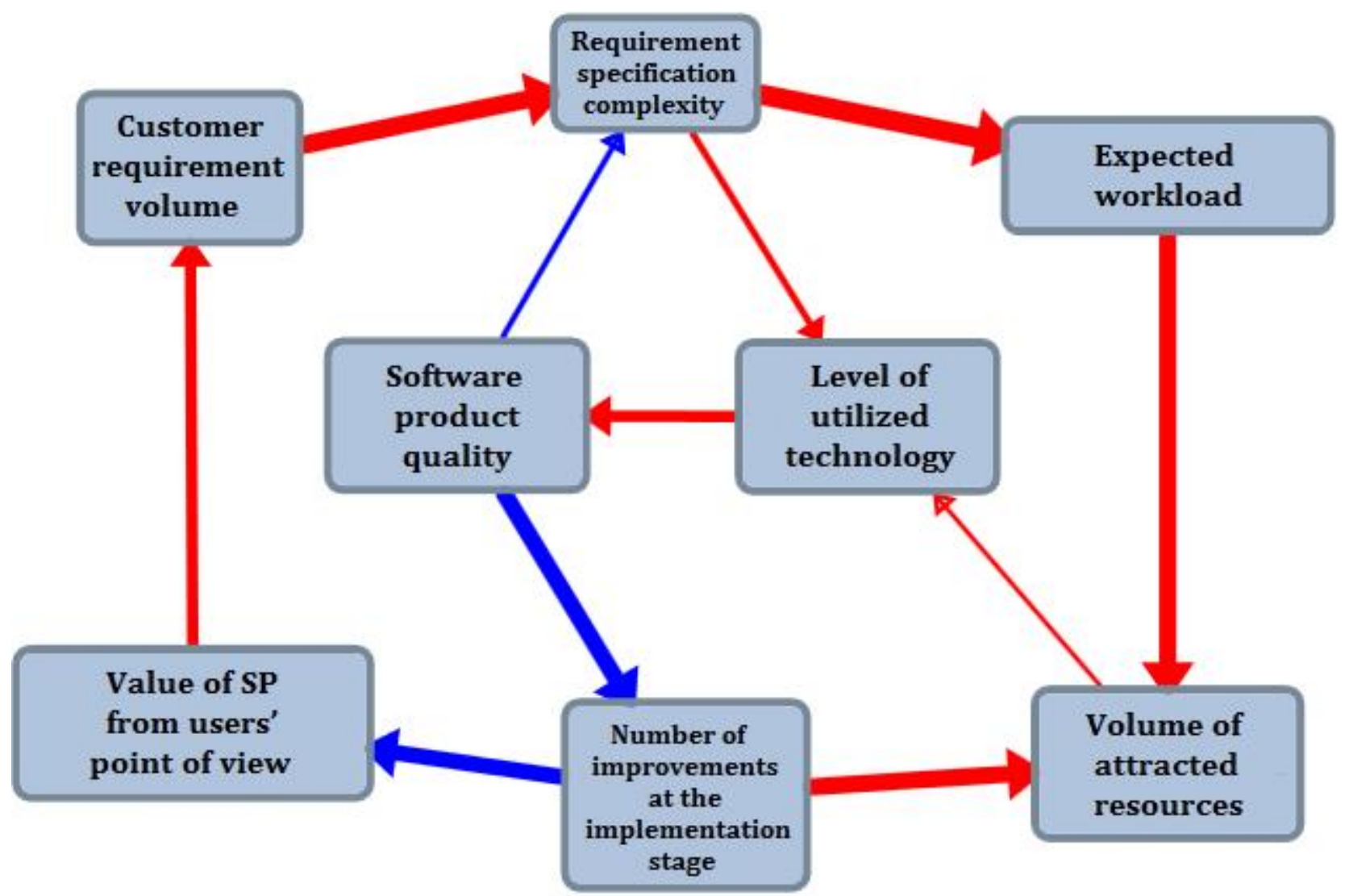

Fig. 9. FCM visual image

The key effect of applying the proposed FCM visualization metaphor for visual analysis of cognitive models is a significant reduction in the time spent on building FCM visual image with a high level of cognitive clarity. So, for the FCM considered, the use of metaphor made it possible to reduce the time required to build such a visual image (Fig. 9) by 4 times.

The resulting visual image can be adapted for visual analysis at any stage of cognitive modeling by using the appropriate representation metaphor (this issue was discussed in detail in the authors' paper [3]). So, the use of the metaphor shown in Fig. 10 allows the analyst to interpret FCM system indicators at the stage of structure and target analysis. Meanwhile, the time spent is reduced by an average of 3-4 times compared to the traditional method of interpretation (implying the need for the analyst to read a large amount of data in numerical representation). Thus, it can be said that the efficiency of cognitive interpretation of FCM visual image increases by means of reducing the time spent on it [11]. 


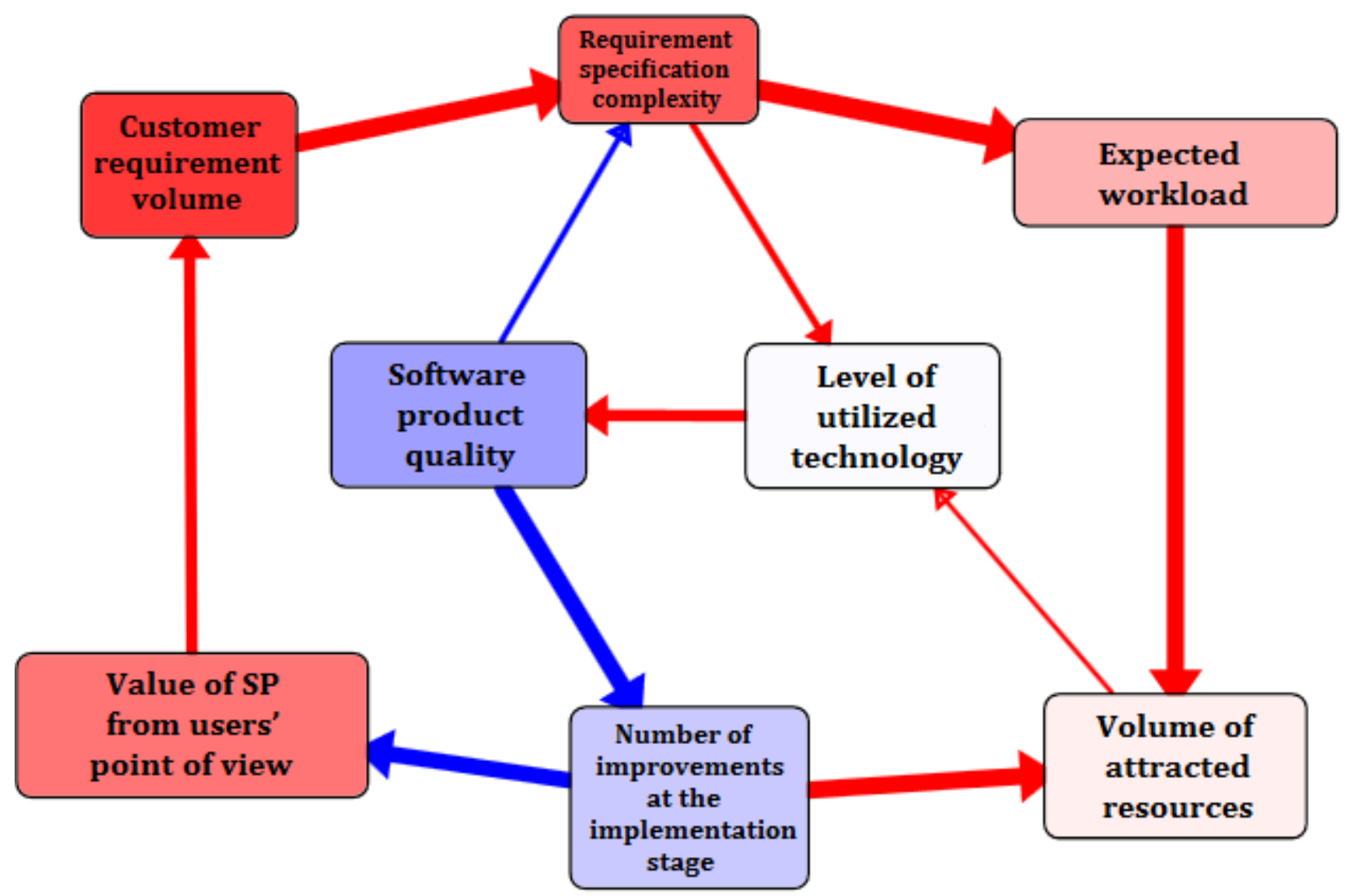

Fig. 10. The result of applying the metaphor of FCM system indicators

\section{Conclusion}

The paper presents the development of the visualization metaphor of fuzzy cognitive maps in the direction of automating the construction of a visual image of a fuzzy cognitive map optimal from the point of view of cognitive clarity criteria. A generalized FCM visualization algorithm using a metaphor is presented. The work of the algorithm is based on the use of graph visualization algorithms and taking into account formalized cognitive clarity criteria. Possible methods of formalizing several of these criteria, which are nontrivial, are described. A decision rule is also proposed for choosing the optimal visual image, which allows for controlling the relative importance of cognitive clarity criteria and determining the acceptable forms of compromise between them. An example of applying the proposed metaphor is given, confirming its efficiency and effectiveness.

Let us indicate directions for further research.

The first one is the search and formalization of relationships between the spatial metaphor and the FCM representation metaphor. In particular, identifying situations in which a change of the representation metaphor requires adjusting preferences according to cognitive clarity criteria, which leads to a change of the optimal visual image.

The second one is the development of recommendations on the selection of parameters of the decision rule proposed in the work based on identifying the relationships between the parameters of this rule and its effectiveness in constructing visual images of various FCMs.

The third one is the development of new decision rules for choosing the optimal FCM tiling from the point of view of cognitive clarity criteria, allowing for more flexible consideration of user's preference characteristics and acceptable forms of compromise among the cognitive clarity criteria.

The reported study was funded by RFBR, project number 19-07-00844. 


\section{References}

1. Borisov V.V., Kruglov V.V., Fedulov A.S. Nechetkie modeli i seti [Fuzzy Models and Networks]. - M.: Goryachaya Liniya - Telekom, 2012. - 284 p. [in Russian]

2. Silov V.B. Prinyatie strategicheskih reshenij v nechetkoj obstanovke [Strategical Decision Making in Fuzzy Environment] - Moscow: INPRO-RES, 1995. - 228 p. [in Russian]

3. Podvesovskii A.G., Isaev R.A. Visualization Metaphors for Fuzzy Cognitive Maps // Scientific Visualization, 2018, Vol. 10, Num. 4, pp. 13-29. - doi: 10.26583/sv.10.4.02

4. Zakharova A.A., Shklyar A.V. Visualization Metaphors // Scientific Visualization, 2013, Vol. 5, Num. 2, pp. 16-24.

5. Meyer B. Self-Organizing Graphs - A Neural Network Perspective of Graph Layout // Whitesides S.H. (eds) Graph Drawing. GD 1998. Lecture Notes in Computer Science, vol 1547. Springer, Berlin, Heidelberg.

6. Noack A. An energy model for visual graph clustering // Proceedings of the 11th International Symposium on Graph Drawing. Springer-Verlag, 2004. - pp. 425-436.

7. Abramova N.A., Voronina T.A., Portsev R.Y. O metodah podderzhki postroeniya i verifikacii kognitivnyh kart $\mathrm{s}$ primeneniem idej kognitivnoj grafiki [Ideas of Cognitive Graphics to Support Verification of Cognitive Maps] // Upravlenie bol'shimi sistemami. Special issue 30.1 "Setevye modeli v upravlenii". - Moscow: ICS RAS, 2010. - pp. 411-430 [in Russian].

8. Huang W., Hong S.H., Eades P. Predicting Graph Reading Performance: A Cognitive Approach // Proc. Asia Pacific Symposium on Information Visualization (APVIS2006). - Tokyo, Japan, 2006. - pp. 207-216.

9. Zakharova A.A., Podvesovskii A.G., Isaev R.A. Matematicheskoe i programmnoe obespechenie podderzhki kognitivnogo modelirovanija slabostrukturirovannyh organizacionno-tehnicheskih system [Mathematical and Software Support for Cognitive Modeling of Semi-structured Organizational and Technical Systems] // International conference CPT2019. - Nizhniy Novgorod: Pub. NNGASU and SRCIPT. 2019. - pp. 131-141 [in Russian].

10. Podvesovskii A.G., Titarev D.V., Isaev R.A. Nechetkie kognitivnye modeli v zadachah analiza i planirovanija programmnyh proektov [Fuzzy cognitive models in software projects analysis and planning] // Vestnik komp'iuternykh i informatsionnykh tekhnologii [Herald of computer and information technologies], 2019, Num. 8, pp. 2231. - doi: 10.14489/vkit.2019.08.pp.022-031

11. Zakharova A.A., Vekhter E.V., Shklyar A.V., Krysko A.V., Saltykova O.A. Quantitative assessment of cognitive interpretability of visualization // Scientific Visualization, 2018, Vol. 10, Num. 4, pp. 145-153. - doi: 10.26583/sv.10.4.11 